\title{
Standardization of intralesional meglumine antimoniate treatment for cutaneous leishmaniasis
}

\author{
Maria Cristina de Oliveira Duque ${ }^{[1],[2]}$, Érica de Camargo Ferreira e Vasconcellos ${ }^{[3]}$, \\ Maria Inês Fernandes Pimentel[ ${ }^{[3]}$, Marcelo Rosandiski Lyra ${ }^{[3]}$, \\ Sandro Javier Bedoya Pacheco ${ }^{[4]}$, Mauro Celio de Almeida Marzochi ${ }^{[3],[5]}$, \\ Cláudia Maria Valete Rosalino ${ }^{[3],[6],[7]}$ and Armando de Oliveira Schubach ${ }^{[3],[5],[8]}$
}

\begin{abstract}
[1]. Secretaria Municipal de Saúde de Timóteo, Timóteo, Minas Gerais, Brasil. [2]. Programa de Pós-Graduação Stricto Sensu em Pesquisa Clínica, Instituto Nacional de Infectologia Evandro Chagas, Fundação Oswaldo Cruz, Rio de Janeiro, Brasil. [3]. Laboratório de Pesquisa Clínica e Vigilância em Leishmanioses, Instituto Nacional de Infectologia Evandro Chagas, Fundação Oswaldo Cruz, Rio de Janeiro, Brasil. [4]. Departamento de Epidemiologia e Métodos Quantitativos em Saúde, Escola Nacional de Saúde Pública Sérgio Arouca, Fundação Oswaldo Cruz, Rio de Janeiro, Brasil. [5]. Programa de Produtividade em Pesquisa, Conselho Nacional de Desenvolvimento Científico e Tecnológico, Brasília, Distrito Federal, Brasil. [6]. Departamento de Otorrinolaringologia e Oftalmologia, Faculdade de Medicina, Universidade Federal do Rio de Janeiro, Rio de Janeiro, Brasil. [7]. Programa Jovem Cientista do Nosso Estado, Fundação Carlos Chagas Filho de Amparo à Pesquisa do Estado do Rio de Janeiro, Rio de Janeiro, Brasil. [8]. Programa Cientista do Nosso Estado, Fundação Carlos Chagas Filho de Amparo à Pesquisa do Estado do Rio de Janeiro, Rio de Janeiro, Brasil.
\end{abstract}

\begin{abstract}
Introduction: Intralesional treatment for cutaneous leishmaniasis has been applied for over 30 years at the Oswaldo Cruz Foundation, Rio de Janeiro, with good therapeutic results and without relevant systemic toxicity. Methods: Meglumine antimoniate was injected subcutaneously, using a long medium-caliber needle (for example, $30 \mathrm{~mm} \times 0.8 \mathrm{~mm}$ ); patients received 1-3 injections, with 15-day intervals. Results: The technique is described in detail sufficient to enable replication. Conclusions: The treatment of cutaneous leishmaniasis with intralesional meglumine antimoniate is a simple, effective, and safe technique, which may be used in basic healthcare settings.
\end{abstract}

Keywords: American cutaneous leishmaniasis. Therapy meglumine antimoniate. Intralesional technique.

For decades, the World Health Organization (WHO) has recommended systemic use of antimonial drugs for treatment of cutaneous leishmaniasis (CL). However, global responses to these treatments are heterogeneous and vary between different species of Leishmania. For example, although administration of $20 \mathrm{mg} \mathrm{Sb}{ }^{5+} \mathrm{kg} /$ day is suggested for 20 days, the treatment regime must follow regional guidelines when available. In 2010, a committee of leishmaniasis specialists updated the global recommendations published in 1990 . The 2010 committee concluded that leishmaniasis treatment was characterized by limited therapeutic options and use of toxic medications. However, CL is not a life-threatening condition, and serious complications of $\mathrm{CL}$, including progression to the mucosal form, are infrequent. Therefore, treatments for CL should not induce life-threatening complications; local treatments are safer and are therefore preferred, despite minimal evidence for their efficacy. However, a risk-benefit analysis of systemic therapy should be conducted for patients

Corresponding author: Dra. Maria Cristina de Oliveira Duque.

e-mail: dermato2@gmail.com

Received 12 June 2016

Accepted 22 August 2016 with complicated or multiple lesions that are very large, or that are located such that they impede local therapy (for example, disfiguring lesions on the face). Therefore, systemic therapy may be indicated after evaluating possible contraindications, provided that adverse effects of such therapies are monitored ${ }^{(1)(2)}$.

Based on the results of recent studies, the Pan American Health Organization (PAHO) updated the WHO recommendations in 2013 and adapted them to both the known characteristics of leishmaniasis and to current health service provision. In addition, the PAHO recommendations emphasize the need to incorporate evidence from national control programs, and to consider variables such as the species of Leishmania, clinical characteristics, and patient access to health services. Ideally, recommendations should be based on controlled clinical trials evaluating the availability of therapeutic alternatives; in particular, trials should be conducted to assess local treatments that currently have low-quality evidence and weak recommendations for use, but which may be effective. The PAHO recommended intralesional treatment (IL) for patients with a single lesion and contraindications to systemic treatment (patients with kidney, liver, and heart diseases, who are $>50$ years of age). IL treatment is contraindicated for patients with immunosuppression and lesions that are $>3 \mathrm{~cm}$ in diameter, or that 
are localized in periarticular areas such as the head. In addition, IL treatment should include 1-5 intradermal infiltrations of $1-5 \mathrm{~mL}$ of meglumine antimoniate (MA) every 3-7 days. The required volume is calculated by the following formula: larger diameter of the lesion in $\mathrm{mm} \times$ smaller diameter $\times 0.008 \mathrm{~mL}^{(3)}$.

In Brazil, systemic therapy with $10-20 \mathrm{mg} \mathrm{Sb}{ }^{5+} / \mathrm{kg} /$ day, to a maximum limit of 3 ampoules daily for 20 days, is the first choice for CL treatment $^{(4)}$. Between 2007 and 2013, 159,301 cases of CL were observed in Brazil; the 885 (0.5\%) associated deaths resulted from either CL or other causes (annual average deaths associated with CL, 126) ${ }^{(5)}$. In 2016, a committee of experts was formed to update the Health Surveillance Secretary of the Ministry of Health [Ministério da Saúde (MS)] manual on tegumentary leishmaniasis. The new version (in preparation) recommends subcutaneous IL treatment for CL.

The National Institute of Infectious Diseases (INI), Oswaldo Cruz Foundation [Fundação Oswaldo Cruz (FIOCRUZ)],
Rio de Janeiro, Brazil, is located in an area of Leishmania (Viannia) braziliensis transmission. Patients at this location typically present with few cutaneous lesions, therefore, patients who cannot receive regular parenteral MA for tegumentary leishmaniasis, or who indicate significant signs of toxicity with systemic antimony, receive IL treatment with subcutaneous MA, despite the different recommendations by the $\mathrm{PAHO}^{(3)}$ and the $\mathrm{MS}^{(4)(6)(7)(8)(9)(10)}$.

In order to provide new evidence for the efficacy of IL treatment with MA, the technique used at the INI/FIOCRUZ is described as follows (Figure 1): 1) apply skin antiseptic around the lesion; 2) infiltrate with MA; if the patient experiences pain, administer an injection of $1 \%$ lidocaine; 3 ) use a long medium-caliber needle (for example, $30 \mathrm{~mm} \times 0.8 \mathrm{~mm}$ ) to facilitate infiltration; 4) use a syringe with a luer-lock connection because high resistance occurs when infiltrating the lesion, leading to abrupt needle undocking with drug extravasation. It may be necessary to use the palm to depress the plunger;
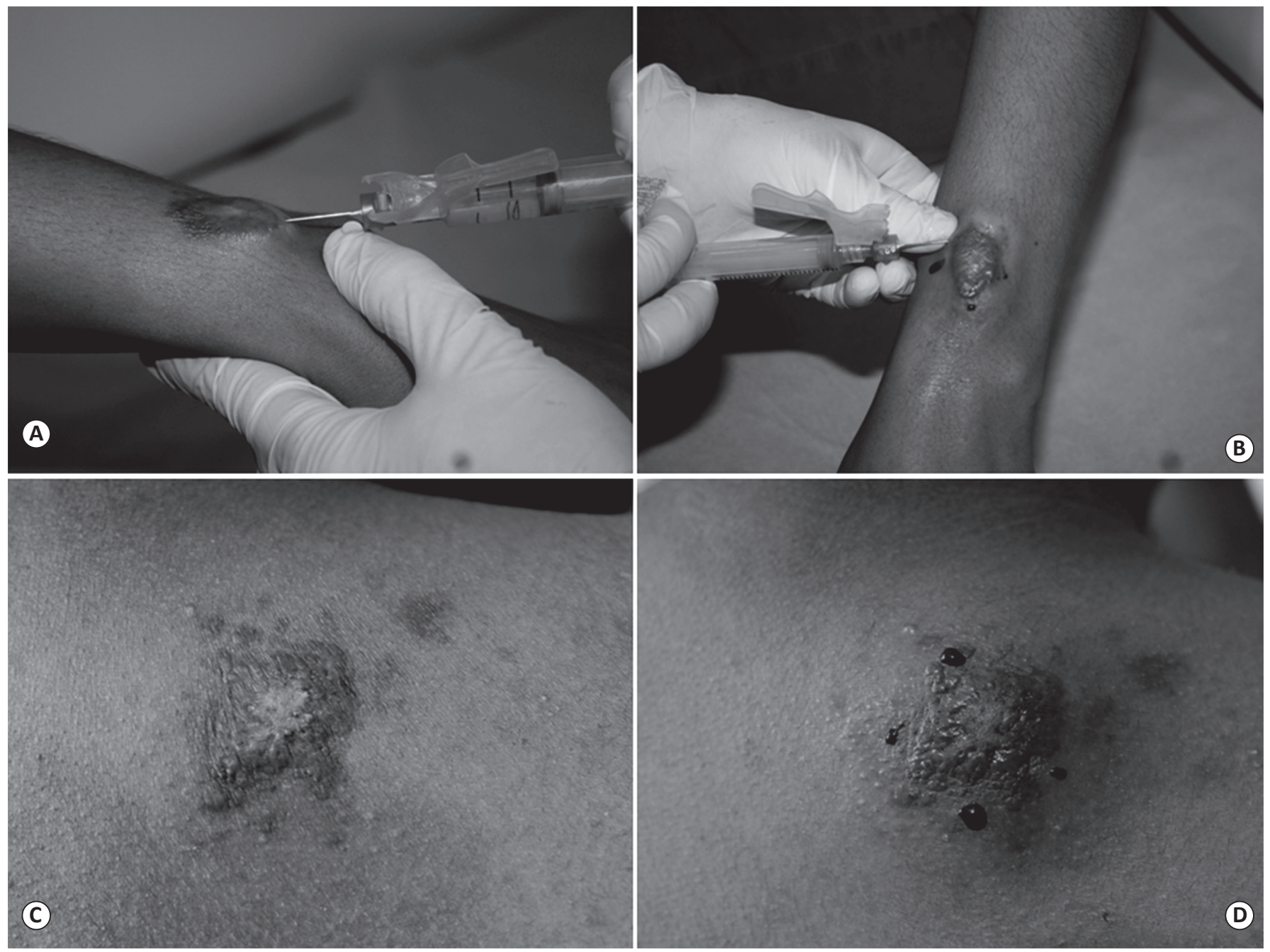

FIGURE 1. Instructions for intralesional treatment of cutaneous leishmaniasis with MA. (A) use a relatively long medium-caliber needle and a luer-lock syringe. Insert the needle into the skin at a $45^{\circ}$ angle, $0.5-1.0 \mathrm{~cm}$ distant from the edge of the lesion; (B) inject MA into the subcutaneous tissue beneath each quadrant and toward the center of the lesion; (C and D) inject the volume needed to infiltrate the base of the lesion by raising it and leaving it intumescent. In practice, the necessary volume of MA can be injected until the infiltrated area is intumesced and acquires a consistency similar to a cheek full of air upon palpation. MA: meglumine antimoniate. 
5) insert the needle into the skin at a $45^{\circ}$ angle, $0.5-1.0 \mathrm{~cm}$ distant from the edge of the lesion, and into the subcutaneous tissue beneath each quadrant, toward the center of the lesion; ensure siphoning before application to avoid injecting intravascularly; 6) inject the volume needed to infiltrate the base of the lesion by raising it and producing intumescence (generally $5-20 \mathrm{~mL}$ ). It is possible to infiltrate small lesions from 1 or 2 points. 7) During the infiltration, jet extravasation of the drug may occur through the surface of the lesion. Therefore, goggles, a face mask, gloves, and an apron are recommended.

Lesions on bone protuberances, such as the pre-tibial region, usually require a lesser volume compared to other lesions. Lesions of the same size, but which are located in regions such as the abdomen or neck require a greater volume. Therefore, calculating the volume of MA to be injected ${ }^{(3)}$ based only on the area of the lesion, is not always useful. In practice, the necessary volume of MA can be injected until the infiltrated area is intumesced and acquires a consistency that is similar to a cheek full of air upon palpation. The maximum daily dose of MA recommended via intramuscular (IM) or intravenous (IV) is $15 \mathrm{~mL}$ in Brazil. However, given the positive therapeutic results obtained with IL volumes $>5 \mathrm{~mL}$, with an efficiency of $83.3 \%$ and without significant systemic toxicity ${ }^{(8)}$, subcutaneous infiltration of greater MA volumes appear to be appropriate. Additionally, we do not contraindicate infiltrating lesions $>3 \mathrm{~cm}$ or those that are located in periarticular areas or in the head; however, the anatomical peculiarities of the face, as well as the presence of vessels, nerves, and noble structures must be considered ${ }^{(3)(4)(6)(7)(8)}$.

A favorable therapeutic response after 1-3 applications is expected. If total epithelialization of the lesions is not observed by approximately 15 days after the first application, a second application is indicated. If total epithelialization is not observed by approximately day 30 , a third and last application is indicated. The patient should then be reexamined every 15 days, and progression towards epithelialization should be observed for as many as 120 days. In the months following epithelialization, crusts, desquamation, infiltration, and erythema disappear successively, and the lesion is healed ${ }^{(7)(8)(9)(10)}$. Therefore, short intervals of 3-7 days between treatments ${ }^{(3)}$ are unnecessary. Furthermore, treatment can be reinitiated using the same protocol if continuous progression to complete healing does not occur.

Adverse clinical, laboratory, and electrocardiographic effects mild or moderate may be observed; however, these effects do not indicate treatment interruption. Local or systemic cutaneous reactions to IM MA should be considered in relation to IL treatment, with regard to the risk of intense local reactions ${ }^{(9)}$.

Treatment of CL with IL MA is simple, efficient, and safe. Furthermore, this treatment protocol can be used in basic healthcare settings. However, current standard practices include restrictions or recommendations that differ from the protocols used at the INI over the last 30 years. Therefore, IL treatment should be queried and investigated in other centers, particularly in basic healthcare settings. We have described our technique in detail, with the intention that this treatment may be replicated in other health centers, thereby contributing to generation of evidence for treatment efficacy.
The broad suggestion of IL treatment for CL under the same conditions of clinical follow-up and monitoring of adverse effects available for the standard systemic treatment may reduce morbidity and mortality related to the therapy for this disease in Brazil.

\section{Acknowledgements}

The authors thank Maurício Naoto Saheki, Mariza de Matos Salgueiro, and Liliane de Fatima Antonio for their technical contributions.

\section{Conflict of Interest}

The authors declare that there are no conflicts of interest.

\section{Financial Support}

This study was partially supported by Fundação Oswaldo Cruz, by Conselho Nacional de Desenvolvimento Científico e Tecnológico, and by Fundação Carlos Chagas Filho de Amparo à Pesquisa do Estado do Rio de Janeiro, Brazil.

\section{REFERENCES}

1. World Health Organization (WHO). Control of Leishmaniases: report of a WHO Expert Committee. World Health Organ Tech Rep Ser 1990; 793:1-158. PMID: 2124015.

2. World Health Organization (WHO). Control of the Leishmaniases. WHO Technical Report Series No.: 949. Geneva: World Health Organization; 2010. 186p.

3. Organización Panamericana de la Salud (OPAS). Leishmaniasis en las Américas: recomendaciones para el tratamiento. Washington: OPAS; 2013. 43p.

4. Ministério da Saúde. Secretaria de Vigilância em Saúde. .Manual de Vigilância da Leishmaniose Tegumentar Americana. Brasília: SVS/ MS; 2010. 180p.

5. Ministério da Saúde. Secretaria de Vigilância em Saúde. Sistema de Informação de Agravos de Notificação (SINANNET). Leishmaniose tegumentar americana: casos confirmados notificados no SINAN-NET por evolução do caso, 2007-2013. Citado 2016 Mar 6. Disponível em: http://tabnet.datasus.gov.br/cgi/ tabcgi.exe?sinannet/cnv/ltabr.def

6. Oliveira-Neto MP, Schubach A, Mattos M, Gonçalves da Costa SC, Pirmez C. Intralesional therapy of American cutaneous leishmaniasis with pentavalent antimony in Rio de Janeiro, Brazil an area of Leishmania (V.) braziliensis transmission. Int J Dermatol 1997; 36:463-468.

7. Ferreira Vasconcellos EC, Pimentel MIF, Valete-Rosalino CM, Lyra MR, Salgueiro MM, Saheki MN, et al. Effectiveness and safety of intralesional treatment with meglumine antimoniate in patients with the cutaneous presentation of American tegumentary leishmaniasis. Rev Soc Bras Med Trop 2010; 43 (suppl 2):52-55.

8. Vasconcellos ECF, Pimentel MIF, Schubach AO, Oliveira RVC, Azeredo-Coutinho RB, Conceição-Silva $\mathrm{F}$, et al. Intralesional meglumine antimoniate for treatment of cutaneous leishmaniasis patients with contraindication to systemic therapy from Rio de Janeiro (2000 to 2006). Am J Trop Med Hyg 2012; 87:257-260.

9. Ferreira Vasconcellos ECF, Pimentel MIF, Valete-Rosalino CM, Madeira MF, Schubach AO. Resolution of cutaneous leishmaniasis after acute eczema due to intralesional meglumine antimoniate. Rev Inst Med Trop São Paulo 2014; 56:361-362.

10. Schubach AO, Conceição-Silva F. Estado da Arte no Tratamento da Leishmaniose Tegumentar Americana no Brasil. In: ConceiçãoSilva F, Alves CA, editors. Leishmanioses do Continente Americano. $1^{\text {st }}$ edition. Rio de Janeiro: Editora FIOCRUZ; 2014. p. 391-412. 